\title{
Effect of Lycium Barbarum Polysaccharides (LBP) on the cognitive function of rats with type 2 diabetic encephalopathy
}

\author{
Liang $\mathrm{ZHAO}^{1}$, Jun $\mathrm{LI}^{2}$, Leilei YU ${ }^{1}$, Huifeng $\mathrm{WANG}^{3}$, Zhaoliang $\mathrm{LI}^{1^{*}}$ (D), Jie YANG ${ }^{{ }^{*}}$
}

\begin{abstract}
This study aims to explore the molecular mechanism of neuronal axonal lesions caused by high glucose, and to investigate the protective effect of Lycium barbarum polysaccharide (LBP) on neuronal axon damage with in vitro models. A rat model of type II diabetic encephalopathy was established, and the rats were treated with LBP for 10 weeks. Morris water maze experiment was used to detect spatial learning and memory ability. Neuronal axon lesions were measured by in vivo neuron tracing experiment. Immunohistochemistry and Western-blot technology were employed to detect protein localization and expression. Combined with in vitro primary neuron culture, total internal reflection fluorescence microscopy was used to detect the effects of high glucose and LBP on neuronal axon transport. PI3K inhibitors LY294002 and an activator of insulin signaling pathway were used to investigate the possible molecular mechanism. In vivo and in vitro experiments showed that in the hippocampus tissue of STZ-induced type II diabetic encephalopathy model rats, neuronal axons were damaged, accompanied by hyperphosphorylation of Tau protein, which subsequently resulted in axonal transport damage and cognition dysfunction. LBP significantly reduced peripheral blood glucose and serum insulin levels in type II diabetic encephalopathy model rats, thereby alleviating peripheral insulin resistance. At the same time, LBP can significantly improve the learning and memory impairment and brain neuron axon pathology in model rats. LY294002 treatment can effectively block the PI3K/Akt signaling pathway. The phosphorylation level of Tau protein in the LY294002+ LBP treatment group is higher than that of the LBP intervention group, while the insulin treatment can effectively reverse the blocking effect of LY294002 on LBPs. LBP can reduce the phosphorylation level of Tau protein by up-regulating the PI3K/Akt/GSK3 $\beta$ signaling pathway, with a protective effect on neuronal axon damage, thereby improving the cognitive function performance of type II diabetic encephalopathy model rats.
\end{abstract}

Keywords: LBP; type II diabetic encephalopathy; PI3K/Akt/GSK3 $\beta$; neuronal axon damage.

Practical Application: LBP on the Cognitive Function

\section{Introduction}

Accounting for $90 \pm 5 \%$ of all diabetes worldwide, type II diabetes (T2D) is characterized by peripheral insulin resistance which will eventually lead to glucose intolerance and hyperglycemia (J.Y. Sung et.al., 2017). Numerous studies have shown that diabetes affects the nervous system. Currently due to the extended life expectancy of T2D patients, cognitive impairment and dementia have become new complications of T2D. Complications of cognitive deficits caused by T2D are also called diabetic encephalopathy. At present, many studies have focused on diabetic peripheral neuropathy, and the pathophysiological mechanism of neuropathy caused by diabetic encephalopathy has received less attention. However, as the survival period of diabetic patients is significantly prolonged, the prevalence of diabetic cognitive dysfunction is bound to increase sharply (Gratuze et al., 2017; Kim \& Feldman, 2015). Therefore, it is extremely urgent to clarify the pathogenesis of diabetic cognitive dysfunction and to find effective prevention and treatment strategies.

LBP can promote the immune function of T, B, CTL, NK and macrophages, promote the production of cytokines such as IL-2, IL-3 and TNF $\beta$, enhance the immune function of tumor-bearing, chemotherapy and radiation-damaged mice, and regulate neuroendocrine immune regulatory network (Muatasim et al., 2018). However, whether LBP has a protective effect in T2D-induced neuronal axonal transport disorder, or whether it can improve the impaired axonal transport, has not yet been reported. Therefore, in this experiment, we used the T2D rat model induced by streptozotocin (STZ) and the primary neuronal insulin resistance model induced by high glucose to systematically clarify the molecular mechanism that LBP can improve the tau hyperphosphorylation and axonal transport disorder through the PI3K/Akt/GSK-3 $\beta$ signaling pathway. 


\section{Methods}

\subsection{Establishment and grouping of in vitro and in vitro models}

\section{Establishment of type II diabetic encephalopathy rat model}

Male Wistar rats, weighing 180 to 200 g, were fed in an SPF animal room, adaptively fed for 2 weeks, high-sugar and high-fat feeding for 4 weeks, then injected with STZ ( $25 \mathrm{mg} / \mathrm{kg}$ ) into the tail vein. One week after the model was established, blood glucose after 12 hours of fasting and 1 hour and 2 hours postprandial blood glucose were measured. The mice with a blood glucose value greater than 11.2 were collected as successful model mice. After the OGTT test, 65 model mice were obtained. Apart from the normal group, 65 mice were randomly divided into the model group, the $50 \mathrm{mg} / \mathrm{kg} \mathrm{LBP}$ dose group, and the $100 \mathrm{mg} /$ $\mathrm{kg}$ LBP dose group. In the 15th week, or the 10 th week after the drug administration, the behavioral experiment was performed.

\section{Establishment of cell models}

Primary cortical neurons were extracted from Sprague-Dawley pregnant mice at the age of 17 days. After 8 days, the primary cortical neurons were used for the experiment. Experiment one groups: A, Control; B, $45 \mathrm{mM}$ glucose treatment for $24 \mathrm{~h}$; C, 20 $\mu \mathrm{M}$ LBP and $45 \mathrm{mM}$ glucose administration for $24 \mathrm{~h}$; D, $40 \mu \mathrm{M}$ LBP and $45 \mathrm{mM}$ glucose administration for $24 \mathrm{~h}$.

Experiment two groups: A, Control; B, $45 \mathrm{mM}$ glucose treatment for $24 \mathrm{~h}$; C, $40 \mu \mathrm{M}$ LBP treatment and $45 \mathrm{mM}$ glucose treatment for $24 \mathrm{~h}$; D, $10 \mathrm{~nm}$ insulin pretreatment for $1 \mathrm{~h}, 45 \mathrm{mM}$ glucose treatment for $24 \mathrm{~h}$; E, $40 \mu \mathrm{M}$ LY294002 pretreatment $30 \mathrm{~min}, 40 \mu \mathrm{M}$ wolfberry polysaccharide treatment and $45 \mathrm{mM}$ glucose treatment for $24 \mathrm{~h}$; F, $10 \mathrm{~nm}$ insulin pretreatment for 1h, 40 HM LY294002 pretreatment for $30 \mathrm{~min}, 45 \mathrm{mM}$ glucose treatment for $24 \mathrm{~h} ; \mathrm{G}, 40 \mu \mathrm{M}$ LY 294002 pretreatment for $30 \mathrm{~min}$, $45 \mathrm{mM}$ glucose treatment for $24 \mathrm{~h}$.

\subsection{Morris water maze test of rat behavior}

According to the Morris water maze test procedure, the operation was repeated for 6 consecutive days, and the escape latency was automatically recorded. Space exploration test: the platform was removed, following the above operation, the stay time in the target quadrant within $60 \mathrm{~s}$ was recorded, and the percentage of time the animal stays in the target quadrant was calculated.

\subsection{In vivo neuron tracing}

$10 \%$ BDA solution was slowly injected into the cortex of the sensorimotor zone on the right. After heart perfusion with $4 \%$ paraformaldehyde solution, the brain tissue was taken out and stored in $4 \%$ paraformaldehyde solution at $4^{\circ} \mathrm{C}$. BDA staining and development: The brain tissue stored in $4 \%$ paraformaldehyde solution is sectioned with a cryostat, and then developed in DAB for 5 minutes. Finally, the slices were taken out, air-dried, dehydrated and sealed for preservation. Pictures were taken with a microscope.

\subsection{Living cell total internal reverse color fluorescence microscopy technology}

According to the instructions, Lipofectamine 2000 was used to transiently transfect pEGFP-NFM into cortical neurons. The cells used for the axon transport study were cultured in fresh medium for 24 hours, and then treated with $45 \mathrm{mM}$ glucose, with or without $40 \mu \mathrm{M}$ LBP, and the cells were observed by evanescent field fluorescence. The exposure time (100-300 ms) and interval time (4-7 s) were adjusted according to the fluorescence intensity of the image. The single time image was viewed, processed and analyzed through TILL vision 4.0 and Photoshop 7.0.

\subsection{Western-blot method to detect various proteins}

According to the instructions, Western blot was employed to detect the Tau protein expression and the PI3K/Akt/GSK3 $\beta$ signaling pathway.

\subsection{Statistical analysis}

Prism 5 (Graph pad, USA) was used for statistical analysis. All data are expressed as mean \pm standard error (Mean \pm S.E.M). One/Two-way ANOVA was performed, followed by Tukey's Post test. $\mathrm{P}<0.05$ was considered statistically different.

\section{Result}

\subsection{Morris water maze test of rat behavior}

The results showed that, compared with the control group, the rats in the model group needed a longer incubation time during the 5 collection periods to reach the platform. It is worth noting that the time to reach the platform gradually shortened after training, especially in the $100 \mathrm{mg} / \mathrm{kg}$ drug administration group. Similarly, the positioning cruise experiment was performed. Statistics showed that the rats in the model group spent longer time to find the platform. However, after drug treatment, the escape latency was significantly reduced. In addition, the space exploration test was performed to evaluate the ability to maintain the platform spatial memory. Compared with the model group, the drug group rats stayed longer in the target quadrant, as shown in Figure 1.

\subsection{Effect of LBP on neuronal axon pathology in diabetic rats}

The axons of rats in the model group showed disordered nerve fiber arrangement, which was characterized by bubble-like, bead-like, leaky and swollen nerve fibers in the axons. Meanwhile, typical axon mutations were observed in the hippocampus, which was significantly reduced in the control group and the administration group, as shown in Figure 2.

\subsection{Effect of LBP high sugar inducing the axon transport of injured neurons}

After high glucose treatment, the proportion of immobile and slow-moving $\rho E G F P-N F M$ particles in neurons increased significantly, but after drug treatment, it was significantly improved, as shown in Figure 3. 


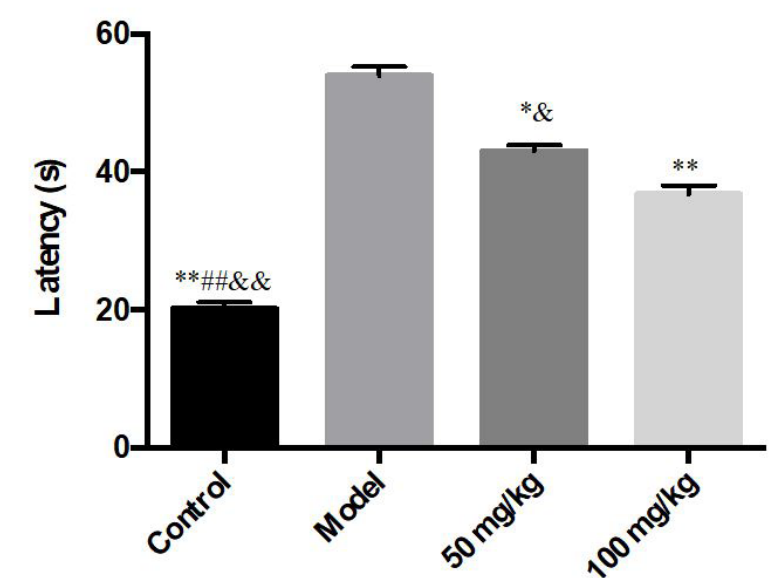

** $\mathrm{p}<0.01$ vs Model $\quad * \mathrm{p}<0.05$ vs Model

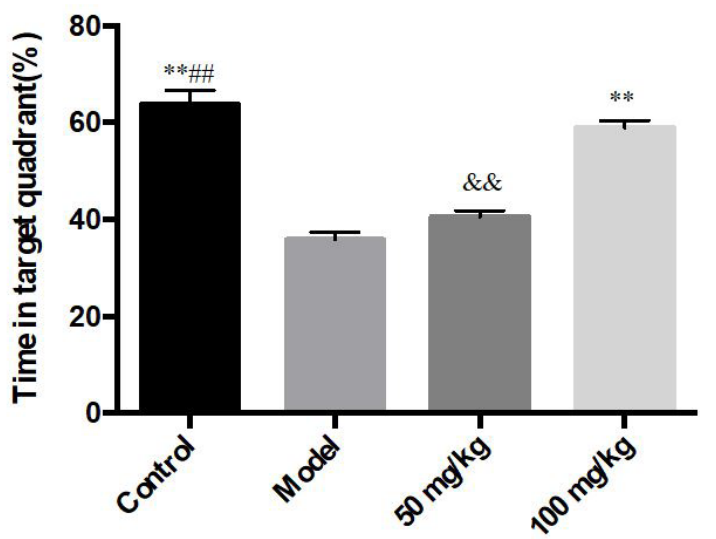

$\# \# \mathrm{p}<0.01$ vs $50 \mathrm{mg} / \mathrm{kg}$

$\& \& \mathrm{p}<0.01$ vs $100 \mathrm{mg} / \mathrm{kg} \quad \& \mathrm{p}<0.05 \mathrm{vs} 100 \mathrm{mg} / \mathrm{kg}$

Figure 1. The effect of LBP on the cognitive function of diabetic rats.
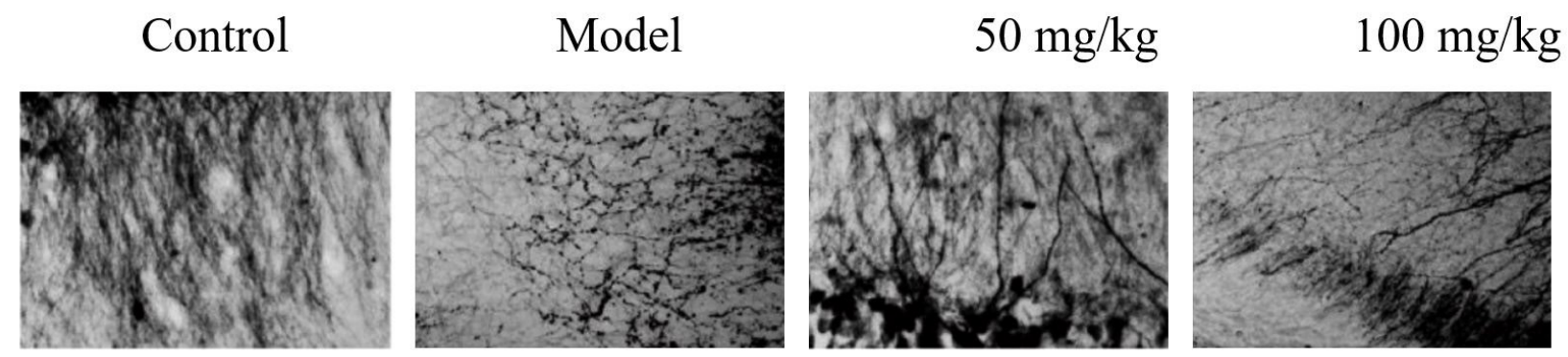

Figure 2. The effect of LBP on neuronal axon pathology in diabetic rats.

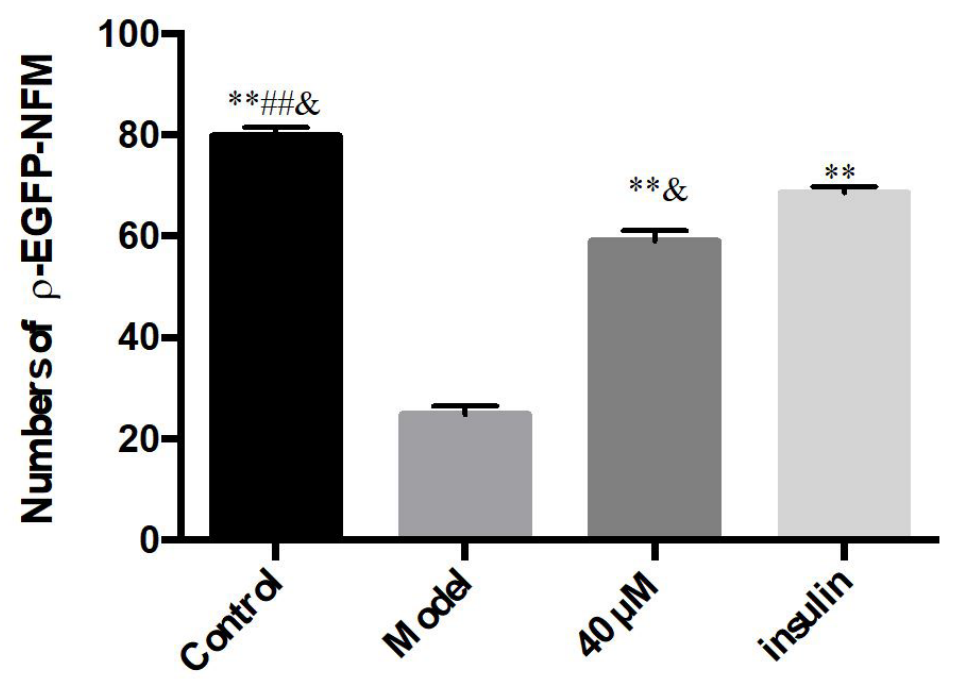

** $\mathrm{p}<0.01$ vs Model \#\# $\mathrm{p}<0.01$ vs $40 \mu \mathrm{M} \quad \& \mathrm{p}<0.05$ vs insulin

Figure 3. The effect of high sugar content of LBP on axon transport in injured neurons. 


\subsection{The effect of LBP on Tau protein in in vivo and in vitro experiments}

The effect of $L B P$ on various proteins of diabetic rats

It was found that in the hippocampus of diabetic rats, the phosphorylation degree of Tau protein Ser 202 and Ser 404 increased by about 2 times. There was no difference in total Tau levels between the groups, indicating that the changes in the Tau protein phosphorylation immunoreactivity were not correlative to changes in the total Tau protein in the hippocampus of the brain, as shown in Figure 4.

The effect of $L B P$ on the proteins in primary neurons induced by high glucose

The drug treatment reduced the hyperphosphorylation of Ser 202 and Ser 404 of Tau protein, indicating that LBP treatment can effectively inhibit the hyperphosphorylation of Tau protein in primary neurons, as shown in Figure 5.

The molecular mechanism of LBP regulating GSK-3ß and Tau protein phosphorylation

LBP participated in the PI3K/Akt/GSK3 $\beta$ signaling pathway to regulate Tau protein phosphorylation. The results showed that pretreatment of neurons with PI3K inhibitor LY294002 can partially block Berberine-induced down-regulated phosphorylation of Tau protein and berberine-activated PI3K/Akt signaling. In addition, after pre-incubation of PI3K inhibitor LY294002, the degree of phosphorylation of Akt and GSK3 $\beta$ induced by LBP and insulin were both inhibited. Therefore, it is suggested that p-Akt and p-GSK3 $\beta$ are the key molecules for the protective effect of LBP, as shown in Figure 6.

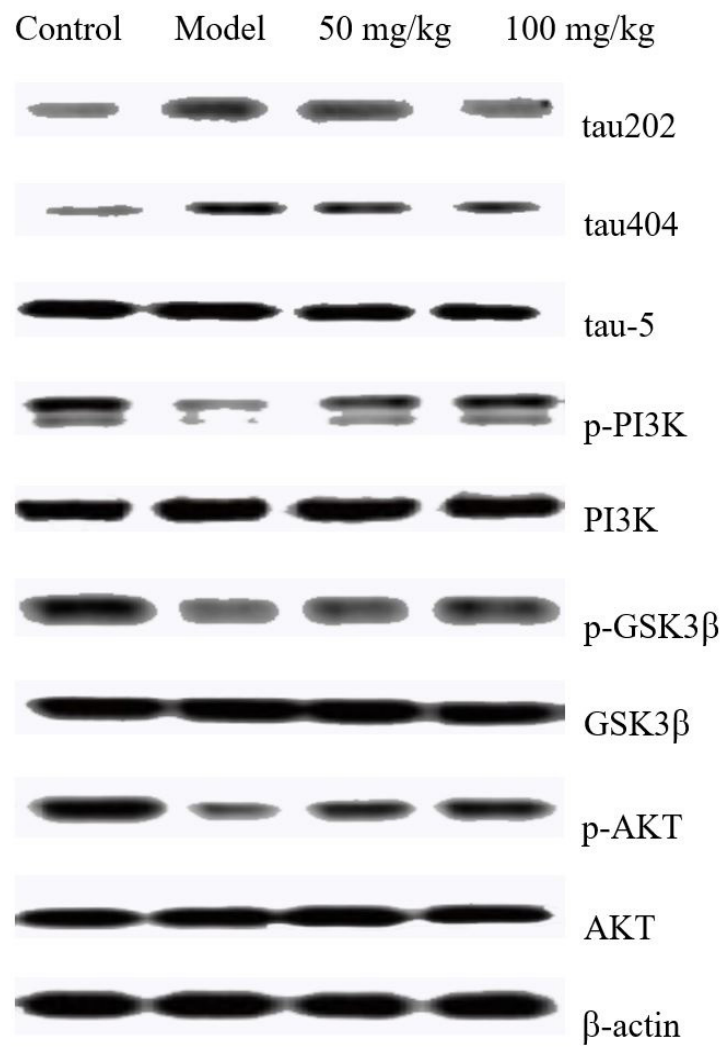

Figure 4. The effect of LBP on various proteins in diabetic rats.

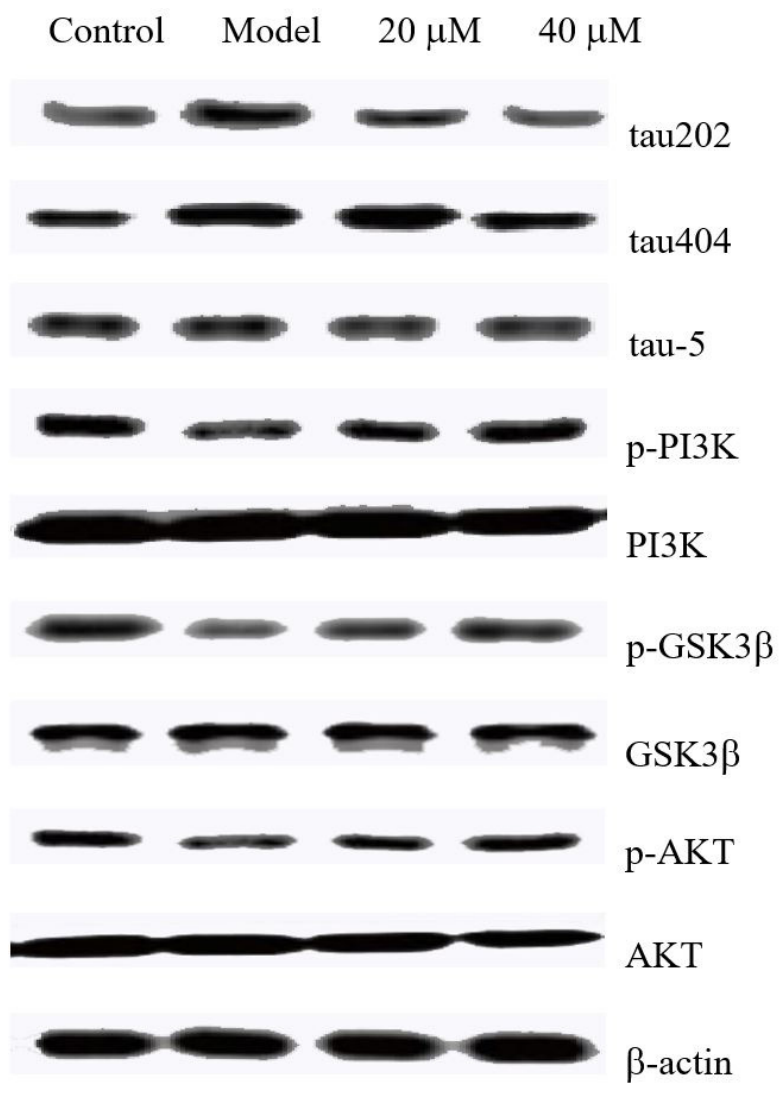

Figure 5. The effect of LBP on the proteins in primary neurons induced by high glucose'.

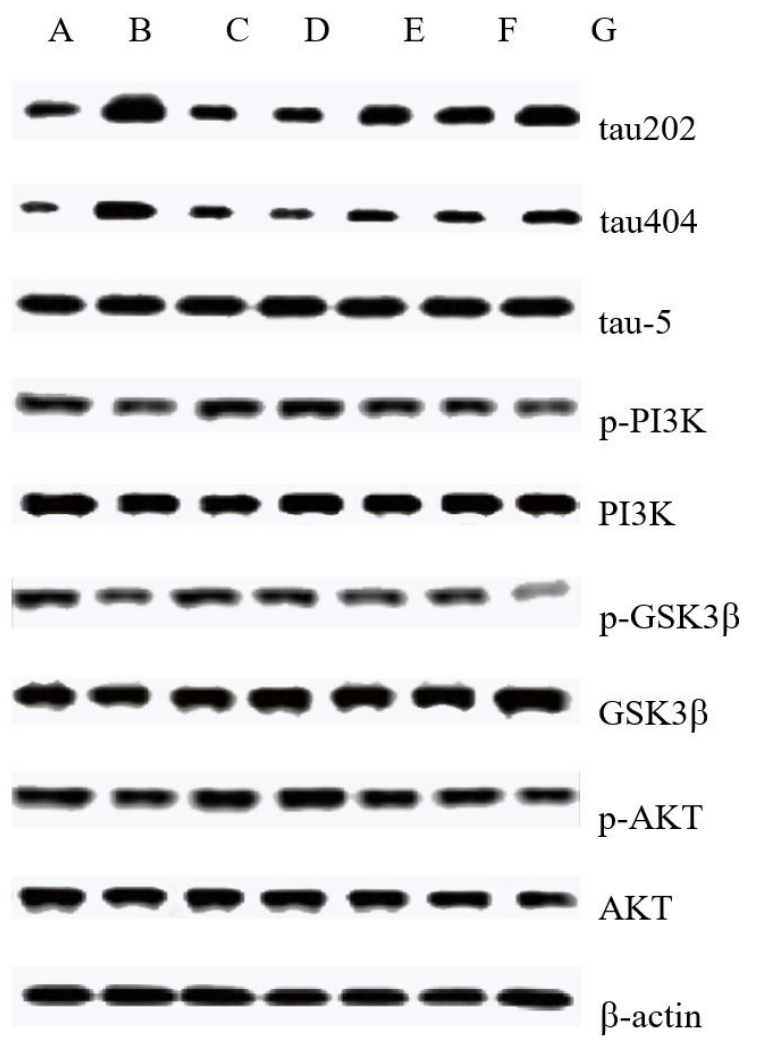

Figure 6. The molecular mechanism of LBP regulating GSK-3 $\beta$ and Tau protein phosphorylation. 


\section{Discussion}

Tau protein binds to microtubules, promotes the assembly of tubulin into microtubules and stabilizes the microtubules of neurons. However, Tau protein hyperphosphorylation can reduce the stability of microtubules and affect microtubule organization. Besides, Tau protein hyperphosphorylation is also considered to be a key target for mediating neuronal degeneration. More and more evidences show that axon pathology caused by hyperphosphorylation of Tau protein leads to diabetes-related cognitive deficits (Huang et al., 2021). Similarly, in animal models and primary neurons, it is also noticed that the degree of Tau protein hyperphosphorylation was positively correlated with glucose levels and cognitive dysfunction. The phosphorylation process can be regulated by the upstream kinase of Tau protein such as GSK-3 $\beta$, which is mainly controlled by the insulin signaling pathway. The down-regulation of insulin signaling in the brain leads to the over-activation of GSK-3 $\beta$, which promotes the hyperphosphorylation of Tau protein (Dey et al., 2017; Chen et al., 2017b; Xu et al., 2017).

Axonal transport refers to the process in which some substances in neurons are transported from the cell body to the axon end or from the axon end to the cell body along the axon. The normal physiological functions of neurons depend on the morphology of axons and dendrites and the normal maintenance of transport functions (Pintana et al., 2017). Axonal transport disorders may impair synaptic plasticity and lead to neurodegenerative diseases (S.A. Hamed, 2017;). More and more evidences indicate that axon diseases, including axon swelling, varicose and axon leakage, represent an early pathogenic characteristic of T2D (Ramos-Rodriguez et al., 2017; Chen et al., 2017a; Yildiz et al., 2021). In addition, site-specific hyperphosphorylation of Tau protein was also detected in the brain of diabetic rats (Bergstrom, 2016). These results together reveal that axonopathy and Tau protein hyperphosphorylation play a key role in the pathogenesis of diabetic encephalopathy. Even in the case of peripheral hyperinsulinemia, down-regulation of the insulin signaling pathway can be observed in the brain of T2D rats, and it is accompanied by the occurrence of neurodegenerative diseases (Sung et al., 2017; Tian et al., 2016). During this process, the kinase GSK-3 $\beta$ is activated, which is a key enzyme in the insulin signaling pathway in the brain of T2D rats and a key upstream kinase of Tau protein (Huang et al., 2017). In addition, studies have shown that intranasal insulin administration can improve the disorder of abnormal insulin signaling and Tau hyperphosphorylation in the brain of diabetic rats, indicating that abnormal insulin signaling in diabetic encephalopathy is closely related to Tau protein hyperphosphorylation.

\section{Conclusion}

In conclusion, the paper clarified the molecular mechanism of LBP through PI3K/Akt/GSK-3 $\beta$ signaling pathway to improve Tau protein hyperphosphorylation and axonal transport disorder, thereby improving the cognitive function of Type II diabetic encephalopathy model rats.

\section{Funding}

Shandong medical and health science and technology development plan project, Project No.: 2018WS159

\section{Reference}

Bergstrom, H. C. (2016). The neurocircuitry of remote cued fear memory. Neuroscience and Biobehavioral Reviews, 71, 409-417. http://dx.doi. org/10.1016/j.neubiorev.2016.09.028. PMid:27693699.

Chen, J. L., Duan, W. J., Luo, S., Li, S., Ma, X. H., Hou, B. N., Cheng, S. Y., Fang, S. H., Wang, Q., Huang, S. Q., \& Chen, Y. B. (2017a). Ferulic acid attenuates brain microvascular endothelial cells damage caused by oxygen-glucose deprivation via punctate-mitochondriadependent mitophagy. Brain Research, 1666, 17-26. http://dx.doi. org/10.1016/j.brainres.2017.04.006. PMid:28438530.

Chen, Q., Mo, R., Wu, N., Zou, X., Shi, C., Gong, J., Li, J., Fang, K., Wang, D., Yang, D., Wang, K., \& Chen, J. (2017b). Berberine ameliorates diabetes-associated cognitive decline through modulation of aberrant inflammation response and insulin signaling pathway in DM rats. Frontiers in Pharmacology, 8, 334. http://dx.doi.org/10.3389/ fphar.2017.00334. PMid:28634451.

Dey, A., Hao, S., Wosiski-Kuhn, M., \& Stranahan, A. M. (2017). Glucocorticoid-mediated activation of GSK3beta promotes tau phosphorylation and impairs memory in type 2 diabetes. Neurobiology of Aging, 57, 75-83. http://dx.doi.org/10.1016/j. neurobiolaging.2017.05.010. PMid:28609678.

Gratuze, M., El Khoury, N. B., Turgeon, A., Julien, C., Marcouiller, F., Morin, F., Whittington, R. A., Marette, A., Calon, F., \& Planel, E. (2017). Tau hyperphosphorylation in the brain of ob/ob mice is due to hypothermia: importance of thermoregulation in linking diabetes and Alzheimer's disease. Neurobiology of Disease, 98, 1-8. http:// dx.doi.org/10.1016/j.nbd.2016.10.004. PMid:27793638.

Hamed, S. A. (2017). Brain injury with diabetes mellitus: evidence, mechanisms and treatment implications. Expert Review of Clinical Pharmacology, 10(4), 409-428. http://dx.doi.org/10.1080/1751243 3.2017.1293521. PMid:28276776.

Huang, M., Duan, J., Yu, B., Zheng, S., Chen, Q., Lin, F., Zeng, N., \& Ling, B. (2021). Clinical value of thalidomide on kk-rat model through TNF-a mediated inflammation approach. Food Science and Technology, [Ahead of print].

Huang, N. Q., Jin, H., Zhou, S. Y., Shi, J. S., \& Jin, F. (2017). TLR4 is a link between diabetes and Alzheimer's disease. Behavioural Brain Research, 316, 234-244. http://dx.doi.org/10.1016/j.bbr.2016.08.047. PMid:27591966.

Kim, B., \& Feldman, E. L. (2015). Insulin resistance as a key link for the increased risk of cognitive impairment in the metabolic syndrome. Experimental \& Molecular Medicine, 47(3), e149. http://dx.doi. org/10.1038/emm.2015.3. PMid:25766618.

Muatasim, R., Ma, H., \& Yang, X. (2018). Effect of multimode ultrasound assisted extraction on the yield of crude polysaccharides from Lycium Barbarum. Food Science and Technology, 38(Suppl 1), 160-166. http:// dx.doi.org/10.1590/1678-457x.14417.

Pintana, H., Apaijai, N., Kerdphoo, S., Pratchayasakul, W., Sripetchwandee, J., Suntornsaratoon, P., Charoenphandhu, N., Chattipakorn, N., \& Chattipakorn, S. C. (2017). Hyperglycemia induced the Alzheimer's proteins and promoted loss of synaptic proteins in advanced-age female Goto-Kakizaki (GK) rats. Neuroscience Letters, 655, 41-45. http://dx.doi.org/10.1016/j.neulet.2017.06.041. PMid:28652187.

Ramos-Rodriguez, J. J., Sanchez-Sotano, D., Doblas-Marquez, A., Infante-Garcia, C., Lubian-Lopez, S., \& Garcia-Alloza, M. (2017). Intranasal insulin reverts central pathology and cognitive impairment in diabetic mother offspring. Molecular Neurodegeneration, 12(1), 57. http://dx.doi.org/10.1186/s13024-017-0198-4. PMid:28768549.

Sung, J. Y., Tani, J., Chang, T. S., \& Lin, C. S. (2017). Uncovering sensory axonal dysfunction in asymptomatic type 2 diabetic neuropathy. 
PLoS One, 12(2), e0171223. http://dx.doi.org/10.1371/journal. pone.0171223. PMid:28182728.

Tian, X., Liu, Y., Ren, G., Yin, L., Liang, X., Geng, T., Dang, H., \& An, R. (2016). Resveratrol limits diabetes-associated cognitive decline in rats by preventing oxidative stress and inflammation and modulating hippocampal structural synaptic plasticity. Brain Research, 1650, 1-9. http://dx.doi.org/10.1016/j.brainres.2016.08.032. PMid:27566063.
Xu, W., Liu, J., Ma, D., Yuan, G., Lu, Y., \& Yang, Y. (2017). Capsaicin reduces Alzheimer-associated tau changes in the hippocampus of type 2 diabetes rats. PLoS One, 12(2), e0172477. http://dx.doi. org/10.1371/journal.pone.0172477. PMid:28225806.

Yildiz, E., Guldas, M., Ellergezen, P., Acar, A. G., \& Gurbuz, O. (2021). Obesity-associated pathways of anthocyanins. Food Science and Technology, 41(Suppl. 1), 1-13. http://dx.doi.org/10.1590/fst.39119. 